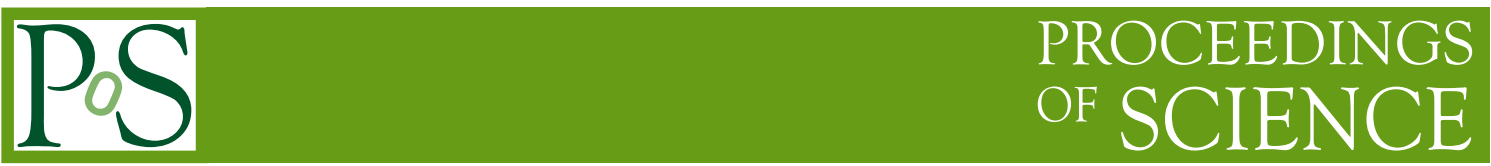

\title{
Difficulties in using GRBs as standard candles
}

\author{
Adam Goldstein*t \\ University of Alabama in Huntsville \\ E-mail: adam.m.goldstein@nasa.gov
}

\begin{abstract}
Gamma-Ray Bursts are isotropically distributed in the observable universe, ranging in comoving distance from a few hundred Mpc to a few thousand Mpc, representing the some of the farthest observable objects in the universe. This large distance coverage is highly attractive to those who study cosmology and the history of the early universe since there are few known objects that represent such a deep and comprehensive representation of the history of the universe. For this reason, there have been extensive studies into the possibility of using GRBs as standard candles much like Type Ia Supernovae, even though little is known about the physical mechanism that produces the observed burst of gamma-rays. We discuss the attempts at defining GRBs as standard candles, such as the search for a robust luminosity indicator, pseudo-redshift predictions, the complications that emission collimation introduces into the estimation of the rest-frame energetics, and the difficulty introduced by the widely varying observed properties of GRBs. These topics will be examined with supporting data and analyses from both Fermi and Swift observations.
\end{abstract}

Gamma-Ray Bursts 2012 Conference-GRB2012,

May 07-11, 2012

Munich, Germany

${ }^{*}$ Speaker.

${ }^{\dagger}$ Supported by NASA GSRP 


\section{Introduction}

Due to their vast range of comoving distances, GRBs have long been sought to be used as standard candles for measuring distances far into the early universe. Several problems exist, however, that prevent GRBs from being cosmological probes in this way. Observationally, the spectral and temporal characteristics of GRBs are distributed over large areas of parameter space. Perhaps these large distributions can be explained by a number of factors, such as the amount of collimation, the Lorentz factor, and even displacement angle of the observer from the center of the jet. Here we enumerate a list of difficulties in using GRBs as standard candles by performing simulations of spectral, temporal, and redshift properties of GRBs. The simulated spectra were drawn from the distribution of spectral parameters corresponding to the GRB Band function contained in the BATSE (Goldstein et al., in prep.) and GBM [1] Spectral catalogs. The temporal properties were drawn from the distribution of $T_{90}$ found in the BATSE 4B GRB catalog [2], while the distribution of redshifts were drawn from the distribution of all known GRBs with spectroscopic redshifts [3].

\section{Luminosity Indicators}

A number of proposed luminosity indicators attempt to use the spectral parameter $E_{\text {peak }}$ as a proxy for the rest-frame luminosity of the burst $[4,5,6,7,8]$. These methods, however, contain a key problem: the photon model-in this case the Band function [9]-is parametrized by $E_{\text {peak }}$, and the flux and fluence, which is used to calculate the rest-frame luminosity or energy, is derived from integrating the photon model over energy. Therefore, there exists a purely mathematical correlation between the $E_{\text {peak }}$ and flux (or fluence), which can lead to an artificial correlation between the rest-frame $E_{\text {peak }}$ and the luminosity or energy. Even assuming a physical correlation between the luminosity or energy and $E_{\text {peak }}$, one must be aware that there is a mixture of artificial correlation due to this complication. In order for such a physical correlation to be ascertained and used in any useful way, it must be determined how much of the observed correlation is artificially introduced.

Using the simulated spectra as well as the data from [4] and the GBM GRBs with known redshift, we can show in Figure 1(a) a plot of the artificially introduced correlation between the rest-frame $E_{\text {peak }}$ and isotropic energy $E_{\text {iso }}$. Overplotting the data and performing a KolmogorovSmirnov test (K-S test), it is found that the hypothesis that the $E_{\text {iso }}$ deduced from the data is drawn from the same distribution as the simulated $E_{i s o}$ can only be rejected at $1 \sigma$ confidence. We can similarly perform the same test if we simulate the Amati relation given the $3 \sigma$ uncertainty in the relation, as in Figure 1(b). In the case of the pure Amati relation, the K-S test shows that the hypothesis that the $E_{i s o}$ values are drawn from the same distribution produced by the Amati relation can be rejected at $92 \%$ confidence. Although not largely conclusive, this indicates a greater chance that the distribution of $E_{i s o}$ values is attributable to the mathematical artifact, rather than the Amati relation by itself. Additionally, comparing the Pearson correlation coefficient of the data $E_{\text {peak }}$ and $E_{i s o}$ to the simulated values, we find $\sim 0 \%$ chance probability that the data correlation is due to the Amati relation, and $\sim 20 \%$ chance probability that the data correlation is due to the mathematical artifact alone.

Similarly, we perform the same correlative analysis using the Yonetoku relation [6], shown in Figures $1(\mathrm{c}) \& 1(\mathrm{~d})$. We find that the connection between the rest-frame $E_{\text {peak }}$ and the isotropic 


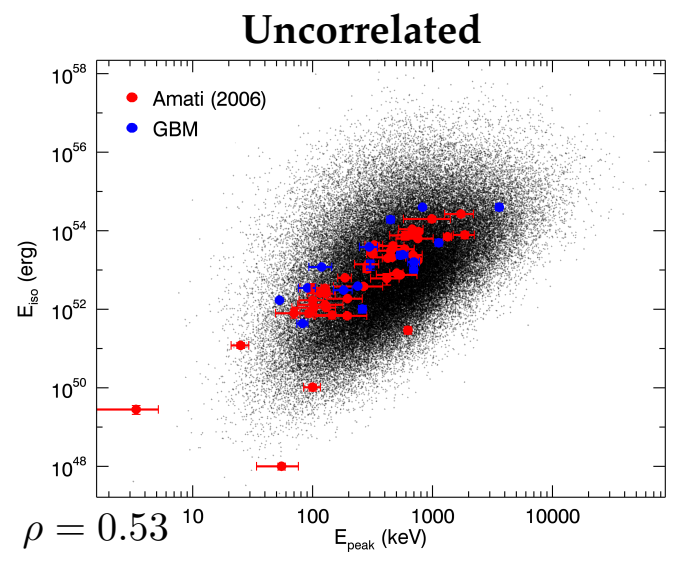

(a)

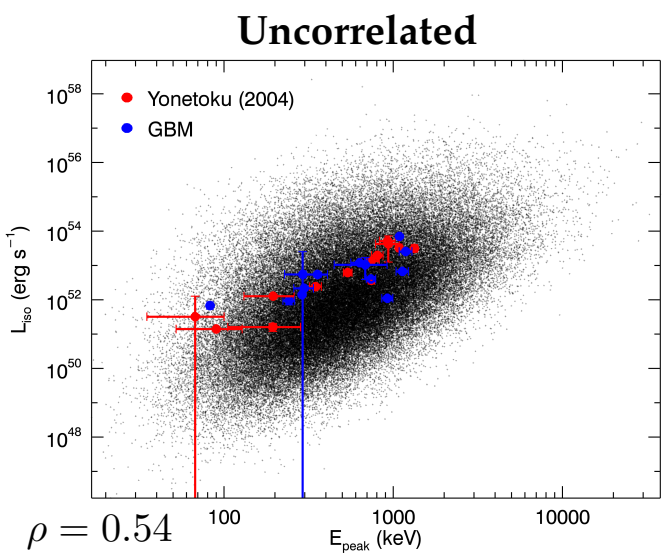

(c)

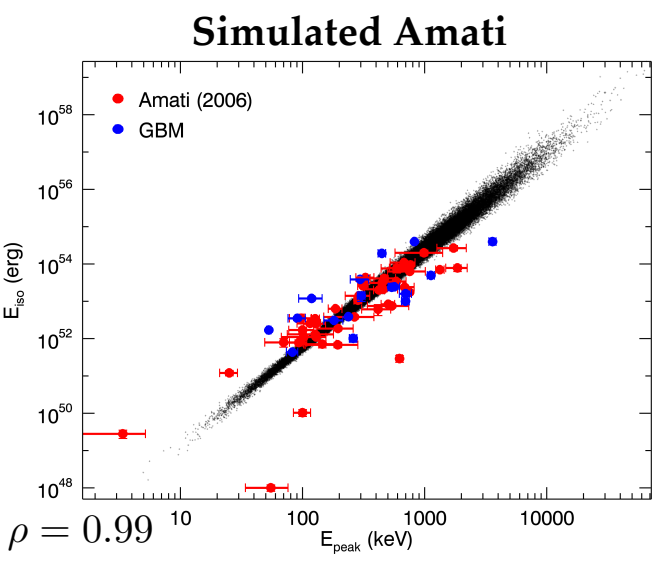

(b)

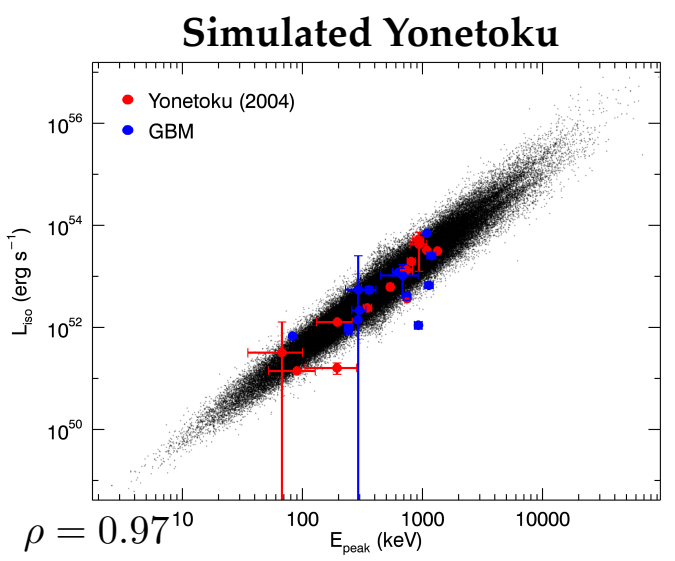

(d)

Figure 1: Simulations depicting the mathematically artificial correlation for 1(a) $E_{\text {peak }}-E_{\text {iso }}$ and 1(c) $E_{\text {peak }}-$ $L_{i s o}$. The corresponding simulated Amati and Yonetoku relations are shown in 1(b) and 1(d). The simulated data are in black, and the observed data are overplotted in red and blue.

luminosity, $L_{i s o}$, has a stronger statistical foundation than what was found for the Amati relation. In this case, the K-S test shows that we can reject the null hypothesis that the data $L_{\text {iso }}$ values are drawn from the simple mathematical artifact at the $2 \sigma$ level. Interestingly, we cannot reject the hypothesis that the data $L_{i s o}$ values are drawn from the Yonetoku relation at more than the $16 \%$ level. The Pearson correlation coefficient of the data shows that the correlation between $E_{\text {peak }}$ and $L_{i s o}$ is supported by only $\sim 5 \%$ chance probability that it is due to the mathematical artifact. Alternatively there is $\sim 0 \%$ chance probability that the data correlation is due to the Yonetoku correlation. This, in addition to the K-S test, indicates that the Yonetoku relation does have some statistical support, although the true correlation between $E_{p e a k}$ and $L_{i s o}$ likely has a larger dispersion than the $3 \sigma$ dispersion assumed in the simulations. 


\section{Estimation of Pseudo-redshifts}

One particular use of GRBs as standard candles is the ability to take an observed quantity and to infer the redshift of the burst from that property. This pseudo-redshift estimation is not only important to study the GRB progenitor formation rate and density, but can serve as an important check on the luminosity relation in question. In particular, we can check how accurately we can recover the redshift of bursts that have spectroscopic estimations as well as examine the limitations of the luminosity relation with respect to the estimation of redshift.

In the case of the Amati relation, we determine that it inherently truncates pseudo-redshift estimates at $\sim 3$ due to the behavior of the relation as a function of redshift $[10,11]$. In addition, when comparing the pseudo-redshift estimates to the spectroscopic redshifts, we find a 0.4 dex dispersion about unity, shown in Figure 2(a). When the uncertainty in parameters is fully propagated to the pseudo-redshift estimation, we determine that for redshifts greater than 2 typically result in uncertainties of approximately half an order of magnitude. This is shown in Figure 2(b). The combination of the poor performance in prediction accuracy and the relatively enormous uncertainties make the Amati relation of little use to producing pseudo-redshifts.

Similarly, the Yonetoku relation can be used to produce pseudo-redshifts, found in Figures 2(c) \& 2(d). We find that for typical values for the best fit index $(\sim-2)$, the pseudo-redshift estimates are not truncated, contrary to the results from the Amati relation. However, when attempting to recover known spectroscopic redshifts of bursts with pseudo-redshifts, we still find a 0.3 dex dispersion about unity. In addition, full propagation of uncertainty leads to an increasing amount of uncertainty as the redshift estimate increases, similar to what was seen with the Amati relation. These results indicate that pseudo-redshifts produced from the Yonetoku relation are not reliable and are of little use when the associated uncertainties are so large.

\section{Jet Collimation}

Another problem with using GRBs as standard candles is caused by the collimation of the relativistic outflow. The true distribution of jet opening angles (and thus the amount of jet collimation) is unknown and the existing distribution of inferred collimation is almost certainly biased toward smaller jet opening angles. Even the method by which jet angles are inferred contains a large amount of uncertainty. This is in large part due to the fact that the estimation of the jet angle depends in large part on the jet break time and the $E_{i s o}$. In fact, there is strictly a power law relation between the collimation-corrected energy and the jet opening angle, but taking into account the uncertainty in the spectral parameters, which affects the uncertainty in $E_{i s o}$, as well as typical uncertainties in the jet break time and circumburst density, the power law relation becomes largely dispersed. This results in over 2 decades of dispersion $(1 \sigma)$ in collimation-corrected energy for a typical jet angle with an uncertainty of $\sim 1^{\circ}$. In addition, jet opening angles are observed to distribute between $\sim 1-10^{\circ}$, but the corresponding collimation-corrected energies for those bursts distribute over 3 decades with no clear correlation with the amount of collimation in the burst. This signals a large difficulty in using GRBs as standard candles since correcting for collimation should naturally produce a clear trend in the burst energies. 


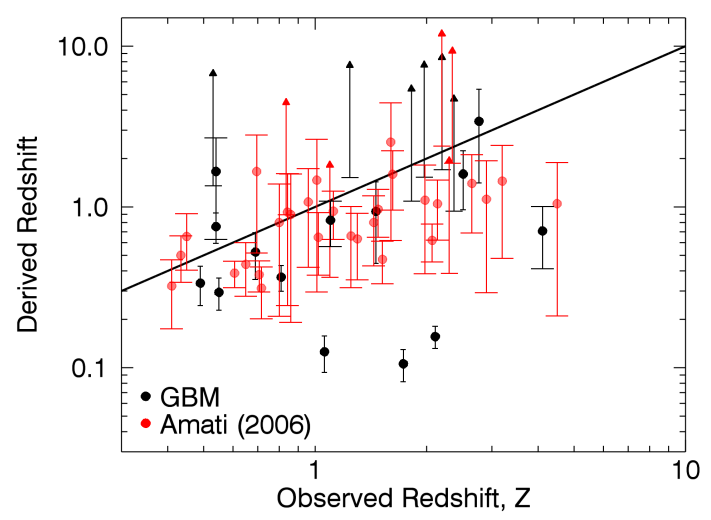

(a)

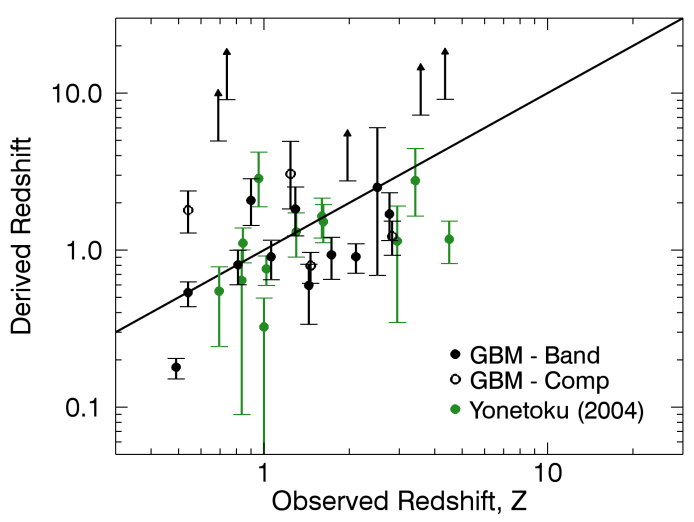

(c)

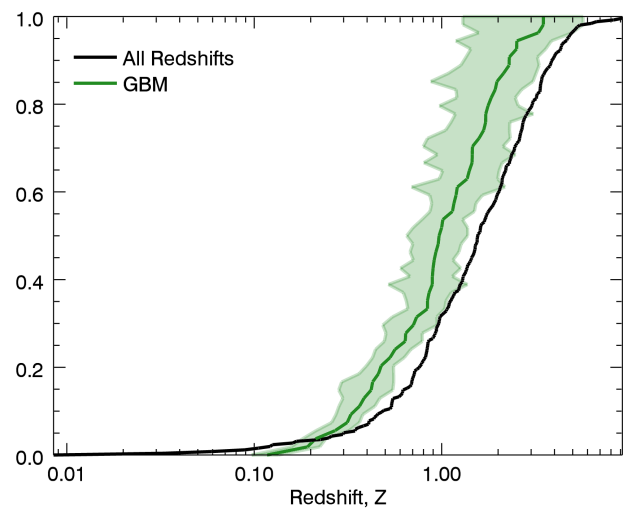

(b)

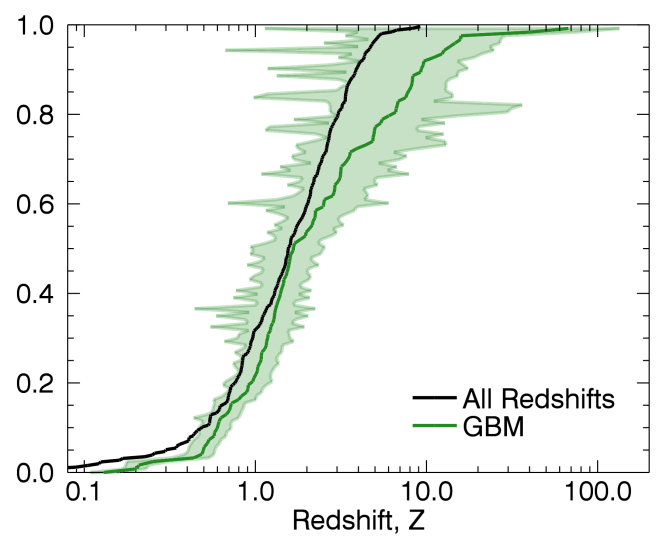

(d)

Figure 2: The pseudo-redshifts plotted against the corresponding spectroscopic redshifts for the Amati relation 2(a) and the Yonetoku relation 2(c). The black lines show a one-to-one correlation. The cumulative distribution of pseudo-redshifts for all GBM bursts best fit by a Band function derived using the Amati relation 2(b) and the Yonetoku relation 2(d). The $1 \sigma$ errors are included in the cumulative distribution to show the relative error increases as a function of the redshift. The black distribution is the cumulative distribution of all GRBs with spectroscopic redshift.

\section{Lorentz Factors}

A contributing factor to the dispersion of observed properties in GRBs is the bulk Lorentz factor of the outflow. For our purposes, we assume the simplest model where the evolution of the Lorentz factor during the prompt emission of the burst does not change significantly and we do not factor the beam profile into the calculation of the Lorentz factor. If we assume that GRBs are standard candles in the rest-frame, we can use the simple relation that $F_{\gamma, o b s} \approx L_{\gamma} \delta^{4}$, where $F_{\gamma, o b s}$ is the observed energy flux in gamma-rays, $L_{\gamma}$ is the luminosity in the rest-frame, which we assume 
to be constant for all GRBs, and $\delta$ is the relativistic Doppler factor. Expanding out, we have

$$
\Gamma\left(1-\sqrt{1-\frac{1}{\Gamma^{2}}}\right) \approx\left(\frac{L_{\gamma}}{4 \pi d_{L}^{2} F_{\gamma, o b s}}\right)^{1 / 4} \sec \theta_{v},
$$

which gives an equation for the Lorentz factor $\Gamma$ in terms of the luminosity, observed energy flux, the luminosity distance, and the viewing angle. Taking the observed energy flux distributions from the BATSE and GBM spectral catalogs, the luminosity distance calculated using the standard cosmology and the current spectroscopic redshift distribution, and a uniform viewing angle distribution, the bulk Lorentz factor distribution can be numerically calculated. Shown in Figure 3, the Lorentz factor distribution assuming GRBs are standard candles spans at least an order of magnitude. Note that this is a best case scenario that is detector-dependent due to the observed flux distribution as well as redshift-biased. Those factors alone could further broaden the Lorentz factor distributions. This key result indicates that even if GRBs are standard candles, as we assumed here, the Lorentz factors can widely disperse observed spectral parameters.

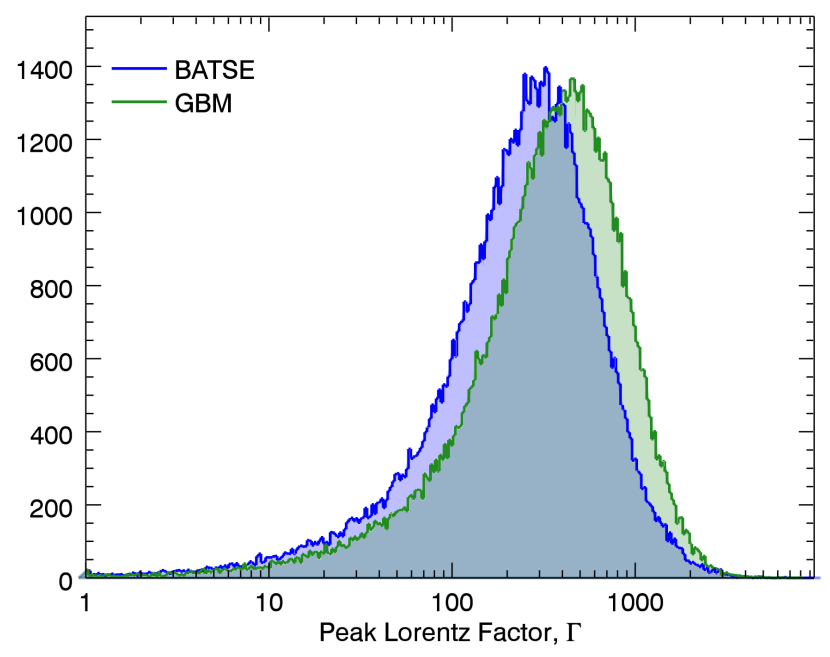

Figure 3: Lorentz factor distributions assuming that GRBs are standard candles in the rest-frame. These distributions are functions of the observed flux, therefore are detector-dependent. The figure shows the difference between the distributions using the BATSE and GBM flux distributions. Note that this optimistic simple case produces distributions that are an order of magnitude wide at full-width half-maximum.

\section{Conclusion}

In conclusion, there are several difficulties facing those who endeavor to use GRBs as standard candles. The luminosity relations that attempt to use $E_{\text {peak }}$ as a proxy for the luminosity fall victim to a mathematical problem that artificially correlates the $E_{\text {peak }}$ with flux and fluence. This artifact convolutes any physical correlation that may exist between the two quantities, and can influence the mean and dispersion of the observed correlation. Using these luminosity relations to estimate pseudo-redshifts can be problematic due to the fact that they do not accurately 
recover spectroscopic redshift values and the uncertainties in the estimates can be quite large, casting doubt on the usefulness of the luminosity relations to calculate redshift. Additionally, jet collimation complicates the estimation of the rest-frame energy and luminosity, especially considering that the calculation of the jet opening angle contains several uncertainties. Correcting for jet collimation does not reveal any trend in energy or luminosity, which could be due to the distribution of Lorentz factors. Optimistically, the Lorentz factors may differ from burst to burst by at least an order of magnitude, which hampers the ability to determine if GRBs are standard candles in the rest-frame. The culmination of all these properties leads to the widely varying properties of GRBs and the difficulties inherent in attempting to use GRBs as standard candles.

\section{References}

[1] A. Goldstein, et al., The Fermi GBM gamma-ray burst spectral catalog: The first two years, ApJS 199 (2012) 19

[2] W. S. Paciesas, et al., The fourth BATSE gamma-ray burst catalog (revised), ApJS 122 (1999) 465

[3] J. Greiner, http://www.mpe.mpg.de/ jcg/grbgen.html

[4] L. Amati, et al., Intrinsic spectra and energetics of BeppoSax gamma-ray bursts with known redshifts, $A \& A 390$ (2002) 81

[5] G. Ghirlanda, G. Ghisellini, \& D. Lazzati, The collimation-corrected GRB energies correlate with the peak energy of their $v F_{v}$ spectrum, ApJ 616 (2004) 331.

[6] D. Yonetoku et al., Gamma-ray burst formation rate inferred from the spectral peak energy-peak luminosity relation, ApJ 609 (2004) 935

[7] B. E. Schaefer, The Hubble diagram to redshift >6 from 69 gamma-ray bursts, ApJ 660 (2007) 16

[8] L. Nava \& G. Ghirlanda, Cosmology with gamma-ray bursts, Mem. S.A.R. It. Suppl. 21 (2012), 82

[9] D. L. Band et al., BATSE observations of gamma-ray burst spectra, ApJ 413 (1993) 281

[10] E. Nakar, \& T. Piran, Outliers to the isotropic energy-peak energy relation in GRBs, MNRAS 360 (2005) L73

[11] D. L. Band \& R. D. Preece, Testing the gamma-ray burst energy relationships, ApJ 627 (2005) 319 